\title{
Co-expression of ERCC1 and Snail is a prognostic but not predictive factor of cisplatin-based neoadjuvant chemotherapy for bladder cancer
}

\author{
ATSUNARI KAWASHIMA ${ }^{1}$, HITOSHI TAKAYAMA ${ }^{1}$, NORIHIKO KAWAMURA ${ }^{2}$, NORITERU DOI ${ }^{3}$, \\ MOTOTAKA SATO $^{1}$, KOJI HATANO ${ }^{1}$, AKIRA NAGAHARA ${ }^{1}$, MOTOHIDE UEMURA ${ }^{1}$, \\ YASUTOMO NAKAI $^{1}$, KENSAKU NISHIMURA ${ }^{2}$, SUSUMU MIYOSHI ${ }^{2}$, KIYOSHI KAWANO ${ }^{3}$, \\ KAZUO NISHIMURA ${ }^{4}$, NORIO NONOMURA ${ }^{1}$ and AKIRA TSUJIMURA ${ }^{1}$ \\ ${ }^{1}$ Department of Urology, Osaka University Graduate School of Medicine, Osaka; \\ Departments of ${ }^{2}$ Urology and ${ }^{3}$ Pathology, Osaka Rosai Hospital, Sakai; \\ ${ }^{4}$ Department of Urology, Osaka Medical Center for Cancer and Cardiovascular Diseases, Osaka, Japan
}

Received December 12, 2011; Accepted February 2, 2012

DOI: $10.3892 / 01.2012 .689$

\begin{abstract}
Neoadjuvant chemotherapy (NC) for bladder cancer has been reported to significantly improve the 5-year survival rate. The aim of the present study was to examine the roles of ERCC1 and Snail in determining the response to chemotherapy in bladder cancer treated with NC and radical cystectomy (RC). The expression of the Snail and ERCC1 proteins was determined by immunohistochemical staining of specimens obtained from 58 patients with bladder tumors treated with $\mathrm{NC}$ and RC. The correlation between clinical response and the expression of Snail and ERCC1 was investigated. Snail and ERCC1 were co-expressed in 24 (41.4\%) of the 58 patients. A marked correlation was found between the expression of Snail and ERCC1 ( $\mathrm{P}=0.001)$. The co-expression of Snail and ERCC1 was not able to predict pathological complete response $(\mathrm{P}=0.202)$. Results of the univariate analysis revealed that the co-expression of Snail and ERCC1 predicted shorter diseasefree survival (DFS) and overall survival (OS) than the negative expression of Snail and/or ERCC1. Moreover, the co-expression of ERCC1 and Snail was the only predictive factor for both DFS $(\mathrm{P}=0.029)$ and $\mathrm{OS}(\mathrm{P}=0.040)$. The expression of Snail was correlated with that of ERCC1 and the co-expression of Snail and ERCC1 was the only significant predictive factor of shorter DFS and OS in patients with bladder cancer treated with NC and RC.
\end{abstract}

Correspondence to: Dr Akira Tsujimura, Department of Urology, Osaka University Graduate School of Medicine, 2-2 Yamadaoka, Suita City, Osaka 565-0871, Japan

E-mail: akitsuji@uro.med.osaka-u.ac.jp

Key words: bladder cancer, ERCC1, Snail, neoadjuvant chemotherapy, co-expression

\section{Introduction}

Bladder cancer is the fourth most common cancer in males in the USA (1). For muscle-invasive bladder cancer, radical cystectomy $(\mathrm{RC})$ and urinary diversion are the gold standard of therapy in many parts of the world (1-3). Neoadjuvant chemotherapy (NC) with cisplatin-based combination chemotherapy significantly improved the 5-year survival rate in two meta-analysis studies $(4,5)$ and pathological complete response (pCR) with NC was correlated with survival $(6,7)$. Although a number of studies have discussed factors which predict pCR or a favorable survival rate (8-10), none of the factors has been proven in a clinical study.

The excision repair cross-complementing group 1 (ERCC1) gene is located on chromosome 19q13.2-q13.3. The ERCC1 protein is crucial in the nucleotide excision repair pathway $(11,12)$. In a previous study, we reported that ERCC1 may predict the prognosis of chemoradiotherapy for bladder cancer and that it was correlated with radiation rather than cisplatin resistance in an in vitro study (13). A correlation between ERCC1 and bladder cancer has been reported (14-17), but whether ERCC1 is capable of predicting a favorable survival rate in patients with advanced bladder cancer treated with cisplatin-based chemotherapy is controversial.

The endothelial-mesenchymal transition (EMT) is significant in invasive bladder cancer $(18,19)$. Mesenchymal markers, including N-cadherin, Zeb1, Snail and Slug, suppress the expression of E-cadherin and are correlated with radiation and cisplatin resistance in numerous types of cancer (20-22). In their study, Hsu et al reported that Snail regulated the expression of ERCC1 and that the co-expression of Snail and ERCC1 was a poor prognostic factor for head and neck cancer treated with cisplatin-based chemotherapy (21). In bladder cancer, however, there have been no studies, to the best of our knowledge, concerning the expression of ERCC1 and Snail as predictors of prognosis for cisplatin-based chemotherapy. 

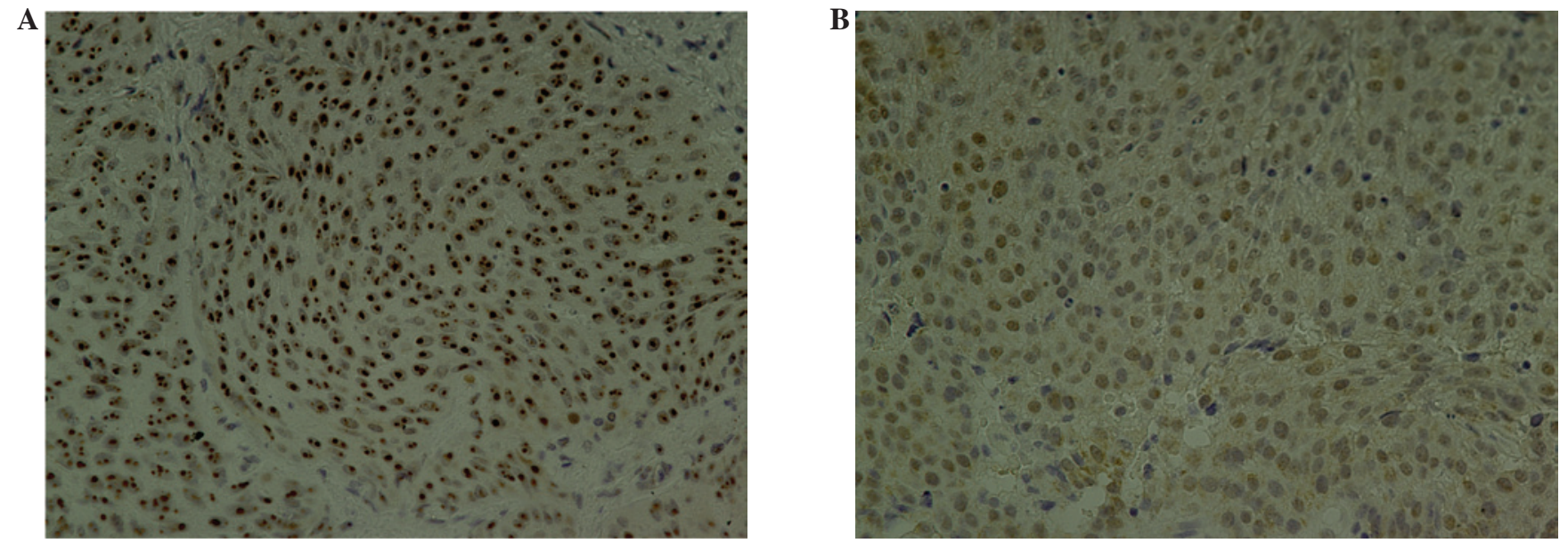

Figure 1. Photomicrographs of paraffin-embedded sections immunohistochemically stained for Snail and ERCC1 in a case of bladder cancer with co-expression of the two markers. (A) Snail-positive bladder cancer. (B) ERCC1-positive bladder cancer.

In this study, we investigated the predictive and prognostic roles of the expression of ERCC1 and Snail to determine the response to chemotherapy in bladder cancer treated with cisplatin-based NC and RC.

\section{Materials and methods}

Patients and samples. In total, 58 patients (50 men, 8 women; median age, 66.0 years; range, $34-78$ years) diagnosed with bladder cancer without organ metastasis and treated with NC and RC at Osaka University or Osaka Rosai Hospital between 1997 and 2010 were enrolled in this study. All 58 patients were clinically staged by computerized tomography (CT) or magnetic resonance imaging (MRI) of the chest, abdomen and pelvis and, following transurethral resection of the bladder tumor, the stage and histological grade of the tumors were determined according to the 5th edition of the TNM classification. Patients were generally followed up postoperatively every 3-4 months for 5 years following RC and every 6 months or annually thereafter. Follow-up consisted of physical examination, routine blood tests, abdominopelvic CT or MRI and chest radiography. Bone scan and chest CT were performed according to the decision of the physician. Approval for this study was obtained from the local institutional review boards of Osaka University and Osaka Rosai Hospital.

Analysis of immunohistochemical staining. Expression of the ERCC1 and Snail proteins was determined by immunohistochemical staining of paraffin-embedded tissue sections of TUR specimens of the bladder tumor just prior to the initiation of NC. Polyclonal anti-ERCC1 antibody was purchased from Santa Cruz Biotechnology, Inc. (\#FL297, Santa Cruz, CA, USA) and the polyclonal anti-Snail antibody was purchased from Abcam (\#ab-63371, Cambridge, MA, USA). Briefly, sections $(5-\mu \mathrm{m})$ were deparaffinized, rehydrated using xylene and alcohol and incubated with $0.3 \% \mathrm{H}_{2} \mathrm{O}_{2}$ to block endogenous peroxidase activity. Prior to ERCC1 immunostaining, antigen retrieval was performed by immersing the sections in $10 \mathrm{mmol} / \mathrm{l}$ citrate buffer ( $\mathrm{pH} 6.0$ ) and boiling in steam for $20 \mathrm{~min}$. Prior to Snail immunostaining, antigen retrieval was performed by immersing the sections in $10 \mathrm{mmol} / 1$ citrate buffer ( $\mathrm{pH}$ 6.0) and antigen retrieval was performed using a pressure chamber (Pascal, Dako, Kyoto, Japan) in which tissues were maintained at $125^{\circ} \mathrm{C}$ for $30 \mathrm{sec}$ and cooled to $90^{\circ} \mathrm{C}$ for $10 \mathrm{sec}$. The sections were then cooled at room temperature for 10 min prior to incubation with the primary antibodies. Immunohistochemistry for ERCC1 and Snail was performed with anti-ERCC1 antibody (1:250 dilution) and anti-Snail antibody ( $1 \mu \mathrm{g} / \mathrm{ml}$ dilution) using the EnVision + detection system (Dako) according to the manufacturer's instructions. Primary antibody was incubated for $60 \mathrm{~min}$ at room temperature and the slides were counterstained with hematoxylin. Two independent investigators (A.K. and H.T.), well-trained in genitourinary pathology and blind to the clinical data, independently evaluated the immunostained slides. ERCC1 nuclear expression was classified into four scoring categories: 0, no expression in tumor cells; $1+$, faint/barely perceptible nuclear expression in $<10 \%$ of tumor cells; $2+$, weak to moderate expression of the entire nucleus in $>10 \%$ of tumor cells; $3+$, strong expression of the entire nucleus in $>10 \%$ of tumor cells. Scores of $2+$ and $3+$ were regarded as positive for ERCC1 staining, as we previously reported (Fig. 1A) (13). Snail nuclear expression was classified using the same four scoring categories as ERCC1 expression. The percentage of positive tumor nuclei was calculated for each specimen and a proportion score was assigned ( 0 if $0 \%, 0.1$ if $1-9 \%, 0.5$ if $10-49 \%$ and 1.0 if $\geq 50 \%$ ). This proportion score was multiplied by the staining intensity of nuclei to obtain a final semi-quantitative H score. Scores of $2+$ and $3+$ were regarded as positive for Snail staining as previously reported (Fig. 1B) (21).

Statistical analysis. The primary outcomes of the patients with bladder cancer were pathological response, overall survival (OS) and disease-free survival (DFS). Estimates of OS and DFS were calculated using the Kaplan-Meier method. The cohorts were defined by age ( $<66$ or $\geq 66$ years), gender (male or female), clinical T stage (T1, T2 or T3, T4), clinical N stage (negative or positive), pathological histology (urothelial carcinoma only or other), pathological tumor grade (grades 1,2 or 3), chemotherapy regimen [methotrexate, Adriamycin, vinblas- 
Table I. Clinical and pathological characteristics of 58 patients with bladder cancer treated with neoadjuvant chemotherapy and radical cystectomy.

\begin{tabular}{|c|c|c|c|c|c|c|c|}
\hline Characteristics & $\begin{array}{c}\text { Total } \\
(\mathrm{n}=58)\end{array}$ & $\begin{array}{l}\text { Snail } \\
\text { positive } \\
(\mathrm{n}=43)\end{array}$ & $\begin{array}{c}\text { Snail } \\
\text { negative } \\
(\mathrm{n}=15)\end{array}$ & $\begin{array}{l}\text { ERCC1 } \\
\text { positive } \\
(\mathrm{n}=25)\end{array}$ & $\begin{array}{c}\text { ERCC1 } \\
\text { negative } \\
(\mathrm{n}=33)\end{array}$ & $\begin{array}{c}\text { Snail and } \\
\text { ERCC1 positive } \\
(\mathrm{n}=24)\end{array}$ & $\begin{array}{c}\text { Snail and/or } \\
\text { ERCC1 negative } \\
(\mathrm{n}=34)\end{array}$ \\
\hline \multirow[t]{2}{*}{ Age, years (median) } & & $\mathrm{P}=0.357$ & & $\mathrm{P}=0.332$ & & $\mathrm{P}=0.254$ & \\
\hline & $34-78(66)$ & $34-78(66)$ & $37-75(63)$ & $42-78(67)$ & $34-77(64)$ & $42-78(68)$ & $34-77$ (64) \\
\hline Gender & & $\mathrm{P}=0.357$ & & $\mathrm{P}=0.671$ & & $\mathrm{P}=0.594$ & \\
\hline Male & 50 & 36 & 14 & 21 & 29 & 20 & 30 \\
\hline Female & 8 & 7 & 1 & 4 & 4 & 4 & 4 \\
\hline Histology & & $\mathrm{P}=0.967$ & & $\mathrm{P}=0.182$ & & $\mathrm{P}=0.157$ & \\
\hline UC only & 54 & 40 & 14 & 22 & 32 & 21 & 33 \\
\hline Others & 4 & 3 & 1 & 3 & 1 & 3 & 1 \\
\hline Clinical T stage & & $\mathrm{P}=0.463$ & & $\mathrm{P}=0.320$ & & $\mathrm{P}=0.096$ & \\
\hline $\mathrm{T} 1$ & 6 & 3 & 3 & 1 & 5 & 0 & 6 \\
\hline $\mathrm{T} 2$ & 13 & 9 & 4 & 6 & 7 & 6 & 7 \\
\hline $\mathrm{T} 3$ & 34 & 27 & 7 & 17 & 17 & 17 & 17 \\
\hline $\mathrm{T} 4$ & 5 & 4 & 1 & 1 & 4 & 1 & 4 \\
\hline Clinical N stage & & $\mathrm{P}=0.507$ & & $\mathrm{P}=0.588$ & & $\mathrm{P}=0.496$ & \\
\hline No & 46 & 35 & 11 & 19 & 27 & 18 & 28 \\
\hline $\mathrm{N}+$ & 12 & 8 & 4 & 6 & 6 & 6 & 6 \\
\hline Highest histology grade & & $\mathrm{P}=0.563$ & & $\mathrm{P}=0.261$ & & $\mathrm{P}=0.118$ & \\
\hline G3 & 42 & 32 & 10 & 20 & 22 & 20 & 22 \\
\hline $\mathrm{G} 2$ & 16 & 11 & 5 & 5 & 11 & 4 & 12 \\
\hline Chemotherapy regimen & & $\mathrm{P}=0.418$ & & $\mathrm{P}=0.730$ & & $\mathrm{P}=0.810$ & \\
\hline $\mathrm{M}-\mathrm{VAC}$ & 50 & 38 & 12 & 22 & 28 & 21 & 29 \\
\hline GC & 8 & 5 & 3 & 3 & 5 & 3 & 5 \\
\hline Chemotherapy response & & $\mathrm{P}=0.074$ & & $\mathrm{P}=0.366$ & & $\mathrm{P}=0.202$ & \\
\hline $\mathrm{CR}$ & 20 & 12 & 8 & 7 & 13 & 6 & 14 \\
\hline Non-CR & 38 & 31 & 7 & 18 & 20 & 18 & 20 \\
\hline ERCC1 expression & & $\mathrm{P}=0.001$ & & & & & \\
\hline Positive & 25 & 24 & 1 & & & & \\
\hline Negative & 33 & 19 & 14 & & & & \\
\hline
\end{tabular}

UC, urothelial carcinoma; CR, complete response; M, methotrexate; V, vinblastine; A, Adriamycin; C, cisplatin; G, gemcitabine.

tine and cisplatin (M-VAC) or gemcitabine and cisplatin (GC)], Snail expression (negative or positive), ERCC1 expression (negative or positive) and co-expression of Snail and ERCC1 (both positive or not both positive). The associations between clinical response status and the clinicopathological characteristics were evaluated using the Fisher's exact test and Pearson's Chi-square test. For the univariate analysis, survival rates were compared according to the clinicopathological parameters previously mentioned. Prognostic factors related to OS and DFS were analyzed with the Cox regression analysis using a step-wise forward selection, with $\mathrm{P}<0.05$ as the criterion for model entry or stay for the multivariate analysis. $\mathrm{P}<0.05$ was considered to indicate a statistically significant result. Statistical analyses were performed using the Statistical Package for the Social Sciences software, version 16.0 (SPSS, Inc., Chicago, IL, USA).

\section{Results}

Patient characteristics. The clinical and pathological characteristics of the 58 patients with bladder cancer in this study are shown in Table I. Fifty-four patients had pure urothelial carcinoma. Histological grade 3 cancer was present in 42 patients and the clinical T stage was T1 in 6 patients, T2 in 13, T3 in 34 and $\mathrm{T} 4$ in 5. Twelve patients had pelvic lymph node metastasis. Fifty patients were treated with M-VAC therapy (median number of courses, 2) and 8 were treated with GC therapy (median number of courses, 2). We assessed the efficacy of NC according to the results of RC. A complete response (CR) was defined as pT0 (no evidence of tumor). Twenty patients achieved CR.

Snail was positively expressed in 43 patients $(74.1 \%)$ and ERCC1 was positively expressed in 25 patients $(43.1 \%)$. With regard to clinicopathological factors, there was no bias 

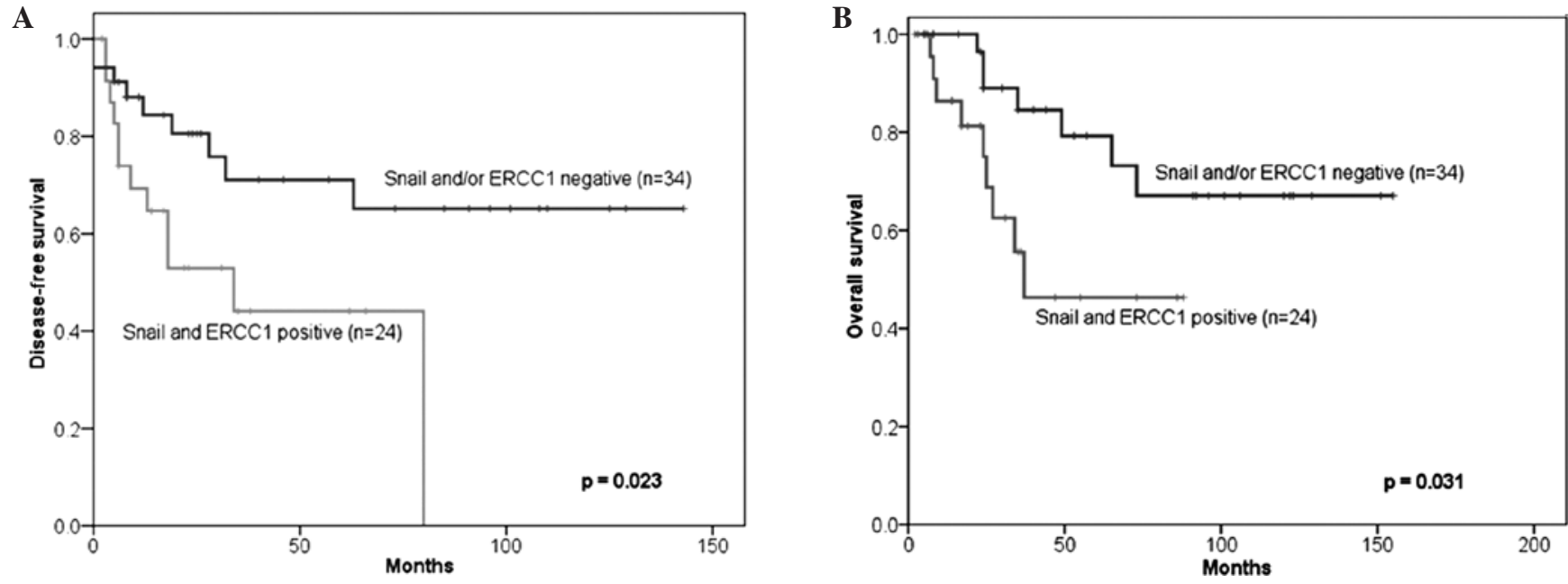

Figure 2. Kaplan-Meier curves of (A) disease-free and (B) overall survival according to Snail and ERCC1 co-expression in the patients treated with cisplatin-based neoadjuvant chemotherapy and radical cystectomy $(\mathrm{n}=58)$.

A

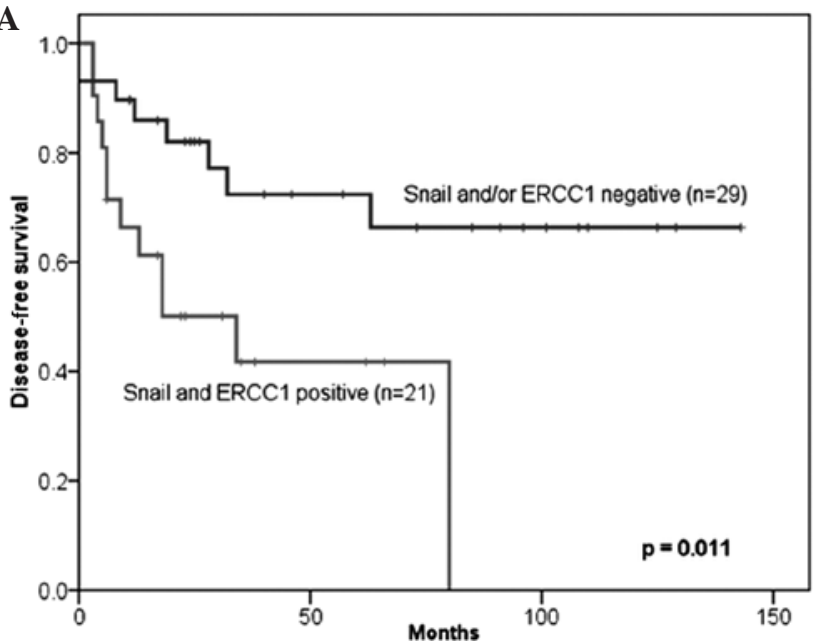

B

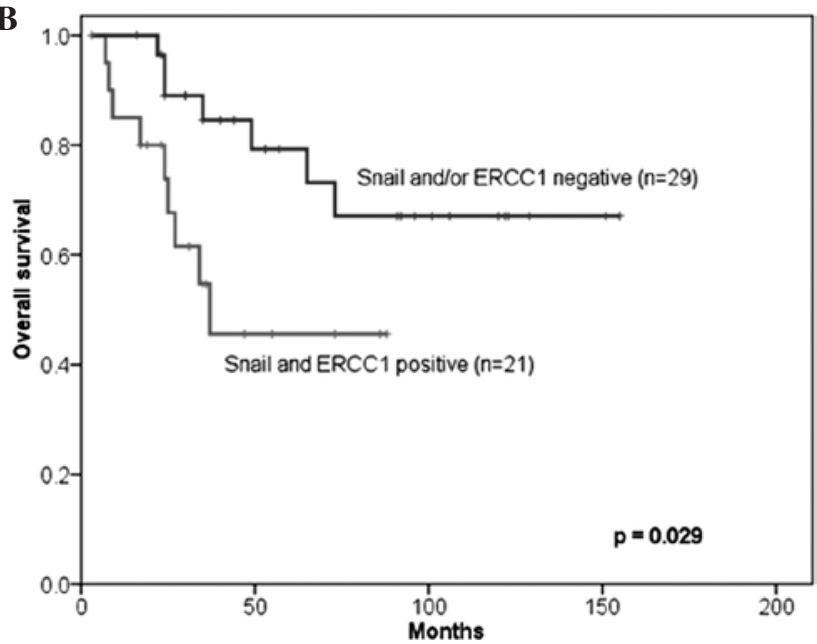

Figure 3. Kaplan-Meier curves of (A) disease-free and (B) overall survival according to Snail and ERCC1 co-expression in the patients treated with M-VAC neoadjuvant chemotherapy and radical cystectomy $(n=50)$. M, methotrexate; $V$, vinblastine; A, Adriamycin; $\mathrm{C}$, cisplatin; $\mathrm{G}$, gemcitabine.

between the patients with a positive and negative expression of both markers. Notably, 24 of the 43 patients (55.8\%) with a positive Snail expression also had a positive ERCC1 expression and 14 patients $(93.3 \%)$ with a negative Snail expression also had a negative ERCC1 expression. A marked correlation was found between Snail and ERCC1 expression $(\mathrm{P}=0.001)$.

The co-expression of Snail and ERCC1 was observed in 24 patients (41.4\%), none of whom was at clinical $\mathrm{T}$ stage T1. There was no bias between patients with and without co-expression. The co-expression of Snail and ERCC1 was not able to predict pCR $(\mathrm{P}=0.202)$. Similarly, the individual expression of either Snail or ERCC1 $(\mathrm{P}=0.074$ and $\mathrm{P}=0.366$, respectively) was not able to predict $\mathrm{pCR}$.

Univariate analysis of predictive factors for DFS and OS. Fiveyear DFS and OS rates were 60.4 and $66.3 \%$, respectively. In patients with a negative Snail expression, 5-year DFS and OS rates were 84.0 and $88.9 \%$, respectively, and those of patients with a positive expression were 51.0 and $58.3 \%$, respectively $(\mathrm{P}=0.019$ and $\mathrm{P}=0.023$, respectively). Five-year DFS and OS rates of patients with a negative expression of ERCC1 were 69.9 and $78.3 \%$, respectively, whereas those of patients with a positive expression were 47.3 and $49.8 \%$, respectively $(\mathrm{P}=0.055$ and 0.070 , respectively). For patients with a co-expression of Snail and ERCC1, 5-year DFS and OS rates were 44.1 and $46.3 \%$, respectively, and the median progression-free survival (PFS) and OS times were 34.0 and 37.0 months, respectively. The co-expression of Snail and ERCC1 more accurately predicted shorter DFS and OS than the negative expression of Snail and/or ERCC1 ( $\mathrm{P}=0.023$ and 0.031 , respectively; Fig. 2). Moreover, in the patients treated only with the M-VAC regimen, the co-expression of Snail and ERCC1 more accurately predicted shorter DFS and OS than Snail and/or ERCC1 negative expression ( $\mathrm{P}=0.011$ and 0.029 , respectively; Fig. 3). Of the remaining clinicopathological factors, only clinical T stage was a significant prognostic factor for longer DFS and no factor predicted the prognosis for OS (Table II).

Multivariate analysis of predictive factors for PFS and OS. Snail expression was a predictive factor for DFS [hazard 
Table II. Univariate and multivariate analysis of predictive factors for DFS and OS in 58 patients treated with cisplatin-based chemotherapy and radical cystectomy.

\begin{tabular}{|c|c|c|c|c|c|c|c|c|}
\hline \multirow[b]{3}{*}{ Prognostic factors } & \multicolumn{4}{|c|}{ DFS } & \multicolumn{4}{|c|}{ OS } \\
\hline & \multicolumn{2}{|c|}{ Univariate } & \multicolumn{2}{|c|}{ Multivariate } & \multicolumn{2}{|c|}{ Univariate } & \multicolumn{2}{|c|}{ Multivariate } \\
\hline & P-value & Exp & $95 \% \mathrm{CI}$ & P-value & P-value & Exp & $95 \% \mathrm{CI}$ & P-value \\
\hline $\begin{array}{l}\text { Age (years) } \\
(<66 \text { vs. } \geq 66)\end{array}$ & 0.404 & - & - & - & 0.410 & - & - & - \\
\hline $\begin{array}{l}\text { Gender } \\
\text { (Female vs. male) }\end{array}$ & 0.842 & - & - & - & 0.542 & - & - & - \\
\hline $\begin{array}{l}\text { Histology } \\
\text { (UC only vs. others) }\end{array}$ & 0.571 & - & - & - & 0.404 & - & - & - \\
\hline $\begin{array}{l}\text { Clinical T stage } \\
(\mathrm{T} 1 / \mathrm{T} 2 \text { vs. T3/T4) }\end{array}$ & 0.027 & - & - & - & 0.117 & - & - & - \\
\hline $\begin{array}{l}\text { Clinical N stage } \\
(\mathrm{N} 0 \text { vs. } \mathrm{N}+)\end{array}$ & 0.664 & - & - & - & 0.867 & - & - & - \\
\hline $\begin{array}{l}\text { Highest histological grade } \\
\text { (Grade } 3 \text { vs. grade } 2 \text { ) }\end{array}$ & 0.250 & - & - & - & 0.668 & - & - & - \\
\hline $\begin{array}{l}\text { Chemotherapy regimen } \\
\text { (M-VAC vs. GC) }\end{array}$ & 0.731 & - & - & - & 0.649 & - & - & - \\
\hline $\begin{array}{l}\text { ERCC1/Snail expression } \\
\text { (ERCC1 and Snail positive vs. } \\
\text { ERCC1 and/or Snail negative) }\end{array}$ & 0.023 & 2.688 & $1.106-6.529$ & 0.029 & 0.031 & 2.864 & $1.050-7.806$ & 0.040 \\
\hline
\end{tabular}

DFS, disease-free survival; OS, overall survival; CI, confidence interval; UC, urothelial carcinoma; M, methotrexate; V, vinblastine; A, Adriamycin; C, cisplatin; G, gemcitabine.

Table III. Univariate and multivariate analysis of predictive factors for DFS and OS in 50 patients treated with M-VAC regimen chemotherapy and radical cystectomy.

\begin{tabular}{|c|c|c|c|c|c|c|c|c|}
\hline \multirow[b]{3}{*}{ Prognostic factors } & \multicolumn{4}{|c|}{ DFS } & \multicolumn{4}{|c|}{ OS } \\
\hline & \multicolumn{2}{|c|}{ Univariate } & \multicolumn{2}{|c|}{ Multivariate } & \multicolumn{2}{|c|}{ Univariate } & \multicolumn{2}{|c|}{ Multivariate } \\
\hline & P-value & Exp & $95 \% \mathrm{CI}$ & P-value & P-value & Exp & $95 \% \mathrm{CI}$ & P-value \\
\hline $\begin{array}{l}\text { Age (years) } \\
(<66 \text { vs. } \geq 66)\end{array}$ & 0.474 & - & - & - & 0.412 & - & - & - \\
\hline $\begin{array}{l}\text { Gender } \\
\text { (Female vs. male) }\end{array}$ & 0.466 & - & - & - & 0.551 & - & - & - \\
\hline $\begin{array}{l}\text { Histology } \\
\text { (UC only vs. others) }\end{array}$ & 0.599 & - & - & - & 0.417 & - & - & - \\
\hline $\begin{array}{l}\text { Clinical T stage } \\
\text { (T1/T2 vs. T3/T4) }\end{array}$ & 0.037 & - & - & - & 0.120 & - & - & - \\
\hline $\begin{array}{l}\text { Clinical N stage } \\
(\mathrm{N} 0 \text { vs. } \mathrm{N}+\text { ) }\end{array}$ & 0.923 & - & - & - & 0.850 & - & - & - \\
\hline $\begin{array}{l}\text { Highest histological grade } \\
\text { (Grade } 3 \text { vs. grade } 2 \text { ) }\end{array}$ & 0.355 & - & - & - & 0.687 & - & - & - \\
\hline $\begin{array}{l}\text { ERCC1/Snail expression } \\
\text { (ERCC1 and Snail positive vs. } \\
\text { ERCC1 and/or Snail negative) }\end{array}$ & 0.011 & 3.108 & $1.237-7.807$ & 0.016 & 0.029 & 2.892 & $1.062-7.876$ & 0.038 \\
\hline
\end{tabular}

DFS, disease-free survival; OS, overall survival; CI, confidence interval; UC, urothelial carcinoma; M, methotrexate; V, vinblastine; A, Adriamycin; C, cisplatin; G, gemcitabine. 
ratio (HR), 4.893; 95\% confidence interval (CI), 1.130-21.192; $\mathrm{P}=0.034]$ but not for OS $(\mathrm{P}=0.053$; data not shown $)$. The co-expression of ERCC1 and Snail was a predictive factor for DFS (HR, 2.688; 95\% CI, 1.106-6.529; P=0.029) and OS (HR, 2.864; 95\% CI, 1.050-7.806; P=0.040; Table II). Moreover, in the patients treated only with the M-VAC regimen, the co-expression of ERCC1 and Snail was also a predictive factor for DFS (HR, 3.108; 95\% CI, 1.237-7.807; P=0.016) and OS (HR, 2.892; 95\% CI, 1.062-7.876; $\mathrm{P}=0.038$; Table III). The co-expression of Snail and ERCC1 was the only significant factor involved in the prediction of shorter DFS and OS by multivariate analysis.

\section{Discussion}

In the present study, we examined the expression of Snail and ERCC1 in bladder cancer and found that the co-expression of Snail and ERCC1 was the only significant factor for predicting prognosis following $\mathrm{NC}$ and $\mathrm{RC}$ against bladder cancer.

ERCC1 is crucial in the nucleotide excision repair pathway and an association of different cancer cell lines with resistance to platinum compounds has been suggested $(11,12)$. In a clinical study using immunohistochemistry, Olaussen et al reported that patients with ERCC1-negative non-small-cell lung cancer appeared to benefit from adjuvant cisplatin-based chemotherapy, whereas patients with ERCC1-positive tumors did not (23). In a clinical study of bladder cancer, a number of studies addressed the correlation between ERCC1 and prognosis (14-17). However, the role of ERCC1 in predicting the prognosis for advanced bladder cancer was controversial. In particular, no studies have discussed whether ERCC1 was capable of predicting CR and prognosis for $\mathrm{NC}$ against bladder cancer. Previously, we reported that ERCC1 might be more resistant to radiation exposure than cisplatin and may be a predictive factor for chemoradiotherapy against muscle-invasive bladder cancer (13). In the present study, the expression of ERCC1 alone was not found to be either a predictive or prognostic factor for NC and RC against bladder cancer by univariate and multivariate analyses (Table I). This result was supported by our earlier in vitro results as the majority of the patients in the present study were not treated with radiation therapy.

EMT has been reported to be significant in invasive bladder cancer $(18,19)$. Snail, one of the markers of EMT, has been reported to predict the intravesical recurrence of superficial bladder cancer (24). However, there have been no studies, to the best of our knowledge, concerning the chemoresistance of Snail in bladder cancer. Thus, we examined Snail expression in bladder cancer patients treated with $\mathrm{NC}$ and $\mathrm{RC}$ and found that Snail was highly expressed in bladder cancer $(74.1 \%)$. This expression may be due to most patients having invasive bladder cancer, although there was no bias between Snail expression and clinical T stage. A negative Snail expression was also a significant predictive factor of longer DFS and OS in the univariate analysis. In the multivariate analysis, however, Snail expression was able to predict longer DFS but not OS. One possible reason for this is that the rate of positive expression of Snail was high. Another is that Snail may be correlated with angiogenesis and occult metastasis. In urothelial carcinoma, Kosaka et al reported that Snail may be correlated with the angiogenesis and prognosis of invasive upper urinary tract carcinoma (25). Therefore, Snail expression may be significant in the prediction of DFS in bladder cancer, as the results of the present study indicate.

Hsu et al have reported that Snail directly regulates ERCC1 expression and that the co-expression of Snail and ERCC1 is a poor prognostic factor in head and neck cancer (21). Therefore, we examined the correlation between the co-expression of Snail and ERCC1 and the prognosis of bladder cancer. Notably, Snail expression was markedly correlated with ERCC1 expression $(\mathrm{P}=0.001$; Table I) and the co-expression of Snail and ERCC1 was a significant prognostic factor to predict longer DFS and OS in univariate and multivariate analysis (Table II). This co-expression was also a significant prognostic factor in the 50 patients treated with M-VAC therapy (Table III).

$\mathrm{NC}$ and $\mathrm{RC}$ have been recommended for invasive bladder cancer $(4,5)$ and it is crucial to be able to predict the CR or prognosis of these therapies. $\mathrm{CR}$ to $\mathrm{NC}$ has been reported to be a significant prognostic factor in several studies $(6,7)$. Takata et al reported that 14 genes were found to be predictive of $\mathrm{pCR}$ and may be prognostic factors in patients with bladder cancer treated with neoadjuvant M-VAC therapy $(10,26)$. With regard to DNA repair genes, BRCA1 mRNA was reported to predict the efficacy of cisplatin-based NC (8). In the present study, although the co-expression of Snail and ERCC1 was not a significant factor for the prediction of CR to NC, notably, the co-expression of the proteins, and not just the individual expression of ERCC1 or Snail, was identified as significant prognostic factors for shorter DFS and OS in the patients treated with a cisplatin-based regimen and also those treated with the M-VAC regimen.

One limitation of the present study is that we did not examine the correlation between Snail and ERCC1 in an in vitro study and did not examine other markers such as ERK1/2, which has been reported to regulate Snail expression (27). Another limitation is that the sample size for immunohistochemical analysis was small and the study was retrospective. More detailed studies are needed in the future to address these limitations.

The results of the present study suggest that the expression of Snail was correlated with that of ERCC1 and the co-expression of Snail and ERCC1 was a significant factor for predicting shorter DFS and OS in bladder cancer treated with $\mathrm{NC}$ and RC. Moreover, Snail may be crucial in the progression of bladder cancer treated with cisplatin-based chemotherapy. Further prospective studies are required to confirm the results of this retrospective study.

\section{Acknowledgements}

This study was supported by a Young Researcher Promotion Grant in 2011 to A. Kawashima.

\section{References}

1. Jemal A, Siegel R, Ward E, Hao Y, Xu J and Thun MJ: Cancer statistics. CA Cancer J Clin 59: 225-249, 2009.

2. Committee for Establishment of the Clinical Practice Guidelines for the Management of Bladder Cancer and the Japanese Urological Association: Evidence-based clinical practice guidelines for bladder cancer (summary - JUA 2009 Edition). Int J Urol 17: 102-124, 2010. 
3. Stenzl A, Cowan NC, De Santis M, et al: The updated EAU guidelines on muscle-invasive and metastatic bladder cancer. Eur Urol 55: 815-825, 2009.

4. Advanced Bladder Cancer (ABC) Meta-analysis Collaboration: Neoadjuvant chemotherapy in invasive bladder cancer: update of a systematic review and meta-analysis of individual patient data. Eur Urol 48: 202-206, 2005.

5. Winquist E, Kirchner TS, Segal R, Chin J and Lukka H: Neoadjuvant chemotherapy for transitional cell carcinoma of the bladder: a systematic review and meta-analysis. J Urol 171: 561-569, 2004

6. Grossman HB, Natale RB, Tangen CM, et al: Neoadjuvant chemotherapy plus cystectomy compared with cystectomy alone for locally advanced bladder cancer. N Engl J Med 349: 859-866, 2003.

7. Matsui Y, Nishiyama H, Watanabe J, et al: The current status of perioperative chemotherapy for invasive bladder cancer: a multiinstitutional retrospective study in Japan. Int J Clin Oncol 10: 133-138, 2005

8. Font A, Taron M, Gago JL, et al: BRCA1 mRNA expression and outcome to neoadjuvant cisplatin-based chemotherapy in bladder cancer. Ann Oncol 22: 139-144, 2011.

9. Pinho MB, Costas F, Sellos J, et al: XAF1 mRNA expression improves progression-free and overall survival for patients with advanced bladder cancer treated with neoadjuvant chemotherapy. Urol Oncol 27: 382-390, 2009.

10. Takata R, Katagiri T, Kanehira M, et al: Validation study of the prediction system for clinical response of M-VAC neoadjuvant chemotherapy. Cancer Sci 98: 113-117, 2007.

11. Gossage L and Madhusudan S: Current status of excision repair cross complementing-group 1 (ERCC1) in cancer. Cancer Treat Rev 33: 565-577, 2007.

12. Park CH, Bessho T, Matsunaga T and Sancar A: Purification and characterization of the XPF-ERCC1 complex of human DNA repair excision nuclease. J Biol Chem 270: 22657-22660, 1995.

13. Kawashima A, Nakayama M, Kakuta Y, et al: Excision repair cross-complementing group 1 may predict the efficacy of chemoradiation therapy for muscle-invasive bladder cancer. Clin Cancer Res 17: 2561-2569, 2011.

14. Bellmunt J, Paz-Ares L, Cuello M, et al: Gene expression of ERCC1 as a novel prognostic marker in advanced bladder cancer patients receiving cisplatin-based chemotherapy. Ann Oncol 18: 522-528, 2007.

15. Hoffmann AC, Wild P, Leicht C, et al: MDR1 and ERCC1 expression predict outcome of patients with locally advanced bladder cancer receiving adjuvant chemotherapy. Neoplasia 12: 628-636, 2010
16. Kim KH, Do IG, Kim HS, et al: Excision repair cross-complementation group 1 (ERCC1) expression in advanced urothelial carcinoma patients receiving cisplatin-based chemotherapy. APMIS 118: 941-948, 2010.

17. Matsumura N, Nakamura Y, Kohjimoto Y, et al: The prognostic significance of human equilibrative nucleoside transporter 1 expression in patients with metastatic bladder cancer treated with gemcitabine-cisplatin-based combination chemotherapy. BJU Int: Dec. 16, 2010 (E-pub ahead of print). doi: 10.1111/j.1464410X.2010.09932.x.

18. Baumgart E, Cohen MS, Silva Neto B, et al: Identification and prognostic significance of an epithelial-mesenchymal transition expression profile in human bladder tumors. Clin Cancer Res 13 1685-1694, 2007.

19. McConkey DJ, Choi W, Marquis L, et al: Role of epithelial-tomesenchymal transition (EMT) in drug sensitivity and metastasis in bladder cancer. Cancer Metastasis Rev 28: 335-344, 2009.

20. Arumugam T, Ramachandran V, Fournier KF, et al: Epithelial to mesenchymal transition contributes to drug resistance in pancreatic cancer. Cancer Res 69: 5820-5828, 2009.

21. Hsu DS, Lan HY, Huang CH, et al: Regulation of excision repair cross-complementation group 1 by Snail contributes to cisplatin resistance in head and neck cancer. Clin Cancer Res 16: 4561-4571, 2010

22. Sayan AE, Griffiths TR, Pal R, et al: SIP1 protein protects cells from DNA damage-induced apoptosis and has independent prognostic value in bladder cancer. Proc Natl Acad Sci USA 106: 14884-14889, 2009.

23. Olaussen KA, Dunant A, Fouret P, et al: DNA repair by ERCC1 in non-small-cell lung cancer and cisplatin-based adjuvant chemotherapy. N Engl J Med 355: 983-991, 2006.

24. Bruyere F, Namdarian B, Corcoran NM, et al: Snail expression is an independent predictor of tumor recurrence in superficial bladder cancers. Urol Oncol 28: 591-596, 2010.

25. Kosaka T, Kikuchi E, Mikami S, et al: Expression of snail in upper urinary tract urothelial carcinoma: prognostic significance and implications for tumor invasion. Clin Cancer Res 16: 5814-5823, 2010

26. Takata R, Katagiri T, Kanehira M, et al: Predicting response to methotrexate, vinblastine, doxorubicin, and cisplatin neoadjuvant chemotherapy for bladder cancers through genome-wide gene expression profiling. Clin Cancer Res 11: 2625-2636, 2005.

27. Ko JC, Su YJ, Lin ST, et al: Suppression of ERCC1 and Rad51 expression through ERK1/2 inactivation is essential in emodinmediated cytotoxicity in human non-small cell lung cancer cells. Biochem Pharmacol 79: 655-664, 2010. 